\title{
§ 18 Das Recht der Älteren im Planungs- und Baurecht
}

Literatur: Bielenberg, Walter/Runkel, Peter/Spannowsky, Willy, Reitzig, Frank, Schmitz, Holger, Raumordnungs- und Landesplanungsrecht des Bundes und der Länder, Band 2, Stand: Mai 2012; Breu, Christian (Hrsg.), Demographischer Wandel und Raumentwicklung in Bayern, Arbeitsmaterial der ARL, Räumliche Konsequenzen des demographischen Wandels, Teil 12, Hannover 2010; Bundesministerium für Verkehr, Bau und Stadtentwicklung (BMVBS) (Hrsg.), Wohnen im Alter, Schriftenreihe Forschungen, Heft 147; Bundesministerium für Verkehr, Bau und Stadtentwicklung (BMVBS)/ Bundesamt für Bauwesen und Raumordung (BBR) (Hrsg.), Kompetenzen und Aufgaben der Raumordnung in der Gestaltung von Kulturlandschaften, BBR-Online-Publikation 19/2007; Bunzel, Arno/ Löhr, Rolf-Peter, Brauchen wir eine neue Baunutzungsverordnung?, ZfBR 2000, S. 307ff.; Eichener, Volker, Die Zukunft der Wohnungspolitik liegt im Bestand, WuM 1999, S.96ff.; Ernst, Werner/ Zinkahn, Willy/Bielenberg, Walter/Krautzberger, Michael, BauGB Kommentar, Lfg. 95, April 2010; v. Franckenstein, Georg, Zur bauplanungsrechtlichen Zulässigkeit von Pflegeheimen im Lichte des Pflege-Weiterentwicklungsgesetzes, ZfBR 2008, S. 763ff.; Janning, Heinz, Der Ausschluss des zentrenschädigenden Einzelhandels im unbeplanten Innenbereich, BauR 2005, S. 1723ff.; Kersten, Jens, Daseinsvorsorge und demographischer Wandel - Wie ändert sich das Raum- und Staatsverständnis? RuR 2006, S. 245ff.; Kersten, Jens, Demographie als Verwaltungsaufgabe, Die Verwaltung 40 (2007), S. 309ff.; Kersten, Jens, in: Veränderung von Verfassung und Verwaltung durch Wissen am Beispiel des demographischen Wandels, in: Voßkuhle, Andreas/Schuppert, Gunnar Folke (Hrsg.), Governance von und durch Wissen, 2008, S. 189ff.; Klinge, Werner, Bebauungspläne zur Steuerung zentraler Versorgungsbereiche nach $\S 9$ Abs. 2a BauGB und erste Überlegungen zur Anwendung, BauR 2008, S.770ff.; König, Helmut/Roeser, Thomas/Stock, Jürgen, Baunutzungsverordnung, 2. Aufl. 2003; Manssen, Gerrit, Öffentliches Baurecht, in: Becker, Ulrich/Heckmann, Dirk/Kempen, Bernhard/Manssen, Gerrit, Öffentliches Recht in Bayern, 5. Aufl. 2011, 4. Teil, S. 415ff.; Mitschang, Stephan/Roeper, Katrin, Stadtumbau auf Gewerbe- und Industriearealen - Gibt es Typologien?, ZfBR 2011, S.10ff.; Reichelt, Thomas, Praxisprobleme der Neufassung des $§ 34$ Abs. 3 BauGB: Geplante zentrale Versorgungsbereiche als Schutzgut? Kumulierte Auswirkungen mehrerer geplanter Vorhaben als Zulassungshindernis? BauR 2006, S. 38ff.; Reidt, Olaf, Die Sicherung zentraler Versorgungsbereiche durch aktive Bauleitplanung - §9 Abs. 2a BauGB und andere Möglichkeiten, BauR 2007, S. 2001ff.; Schmitz, Holger, Die Sicherung zentraler Versorgungsbereiche und der verbrauchernahen Versorgung, ZfBR 2007, S. 532ff.; Schröer, Thomas/Kulick, Christian, Schritte auf dem Weg zu einem „altersgerechten“ Baurecht. NZBau 2011, S. 90ff.; Söfker, Wilhelm, Das Gesetz zur Neufassung des Raumordnungsgesetzes, UPR 2009, S.161ff.; Spannowsky, Willy/Runkel, Peter/ Goppel, Konrad, Raumordnungsgesetz, Kommentar, 2010; Steiner, Udo, Raumordnungs- und Landesplanungsrecht, in: Steiner, Udo (Hrsg.), Besonderes Verwaltungsrecht, 8. Aufl. 2006, Teil V., S. $693 f f$.

Inhaltsübersicht

I. Allgemeines - 1

II. Die Belange der Älteren im Raumordnungs- und Landesplanungsrecht -6

1. Allgemeines zur Raumordnung und Landesplanung - 6
2. Die Umsetzung von Grundsätzen und Zielen der Raumordnung - 12

III. Belange der Älteren im Städtebaurecht -14

1. Die Belange der Älteren in der Bauleitplanung - 14 
2. Die Belange älterer Menschen im sonstigen allgemeinen Städtebaurecht -16

3. Demographischer Wandel und besonderes Städtebaurecht -19
IV. Belange der Älteren im Bauordnungsrecht -22

V. Fazit -25

\section{Allgemeines}

1 Der demographische Wandel stellt Anforderungen auch an das Bau- und Planungsrecht. ${ }^{1}$ Zwei Aspekte sind grundsätzlich zu unterscheiden: Zum einen ist zu fragen, wie sich die Anforderungen an das Wohnen mit steigendem Alter ändern und ob das geltende Recht hierauf vorbereitet ist. Zum zweiten besteht gegebenenfalls ein Anpassungsbedarf hinsichtlich der Bereitstellung von Daseinsvorsorgeeinrichtungen.

2 Der Wohnraumbedarf älterer Menschen weicht von dem jüngerer zwar ab, es ergeben sich aber keine prinzipiellen, sondern eher graduelle Unterschiede. Der Flächenbedarf einer Seniorenwohnung ist oft geringer, da in typischen Seniorenhaushalten keine Kinder mit aufwachsen. ${ }^{2}$ Die Wohnung selbst darf auch nicht zu groß sein, da insbesondere mit zunehmenden körperlichen Einschränkungen die Bewirtschaftung als zu aufwendig und belastend empfunden wird. ${ }^{3}$ Sie muss gegebenenfalls körperlichen Beeinträchtigungen, die im Alter auftreten können, angepasst werden (rollstuhlgerechte Bäder und Toiletten, barrierefreies bzw. barrierereduziertes Wohnen, barrierefreier oder -reduzierter Zugang zur Wohnung). ${ }^{4} 93 \%$ der über 65-Jährigen leben im „normalen“ Wohnungsbestand und wollen überwiegend auch dort bleiben; nur 7\% wohnen in Sonderwohnformen (Heimen, betreutes Wohnen, Altenwohnungen). Der Anteil der Bewohner von Sonderwohnformen erhöht sich allerdings mit steigendem Lebensalter. ${ }^{5}$ Selbst wenn ältere Menschen auf Pflege angewiesen sind, verbleiben sie überwiegend in ihrer „normalen“ Wohnung. Von den ca. 1,86 Mio. Pflegebedürftigen im Alter von 65Jahren und älter werden ca. zwei Drittel zu Hause versorgt. ${ }^{6}$ Ein besonderes Augenmerk bei der Schaffung von altersgerechtem Wohnraum besteht deshalb darin, den bestehenden Wohnraum altersgerecht umzubauen. Hierfür sind vor allem finanzielle Förderungen nötig. Gleiches gilt für den Bau neuer altersgerechter Wohnungen möglichst in der Nähe der bisherigen Wohnungen, soweit damit das Ziel verfolgt wird, die bisher genutzten Wohnungen für jüngere Bewohner frei zu machen. ${ }^{7}$ Auch hierfür sind vor allem finanzielle Anreizsysteme erforderlich.

1 Siehe allgemein auch BMVBS (Hrsg.), Wohnen im Alter, Schriftenreihe Forschungen, Heft 147.

2 Vgl. Eichener, WuM 1999, S. 96, 99.

3 BMVBS (Hrsg.), Wohnen im Alter, S. 31.

4 Siehe Schröer/Kulick, NZBau 2011, S. 90, 90.

5 BMVBS (Hrsg.), Wohnen im Alter, S. 27.

6 BMVBS (Hrsg.), Wohnen im Alter, S. 27.

7 Eichener, WuM 1999, S. 96, 99. 
Ähnliches gilt für Daseinsvorsorgeeinrichtungen. Statt Kinderspielplätzen, Kin- 3 dergärten, Schulen und Jugendzentren müssen die Kommunen verstärkt Treffpunkte für ältere Leute innerhalb oder außerhalb des öffentlichen Raums schaffen. Bereit stehen müssen medizinische Versorgungsangebote bis hin zu Pflegeeinrichtungen. Einkaufsgelegenheiten sollen auch bei Mobilitätseinschränkungen erreichbar sein. Dem öffentlichen Personennahverkehr kommt für ältere Menschen eine besondere Bedeutung zu. Vor allem daraus ergeben sich besondere Anforderungen an das Bauund Planungsrecht. Während der Wohnungsumbau vor allem finanzielle Förderung verlangt, ist die Bereitstellung einer geeigneten Infrastruktur planungsrechtlich vorzubereiten.

Auch wenn gewisse typische Erfordernisse formulierbar sind, die sich aus dem $\mathbf{4}$ demographischen Wandel ergeben, ist das Spektrum möglicher und sinnvoller Maßnahmen weit und nicht abschließend zu umschreiben. Denn die Änderungen in der Bevölkerungszusammensetzung treffen die verschiedenen Regionen in unterschiedlicher Weise. So finden sich in den neuen Bundesländern und im südlichen Niedersachsen besonders hohe Anteile von über 65-Jährigen in der Bevölkerung. ${ }^{8}$ Die Zunahme des Anteils von älteren Menschen wird mittelfristig in Brandenburg, Mecklenburg-Vorpommern und nördlich von München besonders hoch sein. ${ }^{9}$ In manchen Regionen ist mit einem starken Abnehmen der Bevölkerung insgesamt, verbunden mit einer Überalterung, zu rechnen. Hier wird geographisch ein „Dreieck Düsseldorf, Stralsund, Dresden“ bezeichnet. ${ }^{10}$ In den ländlichen Gegenden in diesem Bereich ist mit einem gewissen Wegbrechen von Daseinsvorsorgeleistungen zu rechnen. Hier ist nicht mehr das Wachstum das Paradigma der Infrastrukturpolitik, sondern die Schrumpfung. ${ }^{11}$ Es müssen deshalb für die einzelnen Regionen von den jeweils zuständigen Planungsträgern die erforderlichen Maßnahmen vorbereitet oder getroffen werden.

Die Anpassung der baulichen Umwelt durch Bau- und Planungsrecht an neue 5 gesellschaftliche Erfordernisse erfolgt aber ohnehin mehrstufig. Zunächst sind die gesetzgeberischen Rahmenbedingungen daraufhin $\mathrm{zu}$ hinterfragen, inwieweit sie erkannten neuen Bedürfnissen Raum geben können. Möglicherweise sind den Planungsträgern oder Verwaltungsbehörden neue gesetzgeberische Spielräume einzuräumen. Meist ist dies aber nicht nötig, weil die gesetzlichen Vorgaben ergebnisoffen sind und den Handlungsspielraum für die notwendigen Maßnahmen bereitstellen.

8 Siehe BMVBS (Hrsg.), Wohnen im Alter, S. 30.

9 BMVBS (Hrsg.), Wohnen im Alter, S. 30.

10 Siehe etwa Kersten, Die Verwaltung 40 (2007), S. 309, 332.

11 Vgl. erneut Kersten, Die Verwaltung 40 (2007), S. 309, 322. 


\section{Die Belange der Älteren im Raumordnungs- und Landes- planungsrecht}

\section{Allgemeines zur Raumordnung und Landesplanung}

6 Neue gesellschaftliche Entwicklungen werden häufig zuerst auf der Ebene des Raumordnungs- und Landesplanungsrechts erkannt und ersten Lösungsansätzen zugeführt. ${ }^{12}$ Raum- und Landesplanung ist die Planung der Nutzung des Raumes aus überfachlicher und überörtlicher Sicht. ${ }^{13}$ Damit kommt es zu einer Koordination der Flächennutzung mit Wirkung für die Fachplanungsträger (Wegeplanung, Krankenhausplanung, Städteplanung). ${ }^{14}$ Die auf dieser Ebene gemachten Vorgaben in Gestalt von Grundsätzen oder Zielen der Raumordnung sind anschließend vor allem von den Gemeinden in die kommunale Bauleitplanung umzusetzen (siehe vor allem $\S 1$ Abs. 4 BauGB). Sie binden weiterhin sonstige öffentliche Stellen (§4 ROG).

7 Das Recht der Raumordnung und Landesplanung befindet sich derzeit in einem starken Umbruch. ${ }^{15}$ Nachdem die frühere Rahmengesetzgebungskompetenz des Bundes entfallen ist, ${ }^{16}$ ist Raumordnungsrecht heute Gegenstand der sog. konkurrierenden Gesetzgebungskompetenz des Bundes (Art. 74 Abs. 1 Nr. 31 GG) mit Abweichungsrecht der Länder nach Art. 72 Abs. 3 S. 1 Nr. 4 GG. ${ }^{17}$ Derzeit gilt im Wesentlichen das Raumordnungsrecht des Bundes, das im Raumordnungsgesetz (ROG) ${ }^{18}$ geregelt ist, unmittelbar auch in den Ländern, da diese vielfach noch keine abweichenden eigenen Landesplanungsgesetze erlassen haben. ${ }^{19}$ Es ist jedoch davon auszugehen, dass die Länder in absehbarer Zeit ihr Recht zur Abweichung von der Bundesregelung entweder durch partielle Abweichungsgesetze oder durch Vollregelungen des eigenen Landesplanungsrechts ausüben werden. Die Rechtslage im Einzelnen ist dadurch sehr unübersichtlich. Es lassen sich jedoch einige allgemeine

12 Siehe vor allem Breu (Hrsg.), Demographischer Wandel und Raumentwicklung in Bayern, Arbeitsmaterial der ARL, Räumliche Konsequenzen des demographischen Wandels, Teil 12.

13 Siehe Steiner, in: Steiner (Hrsg.), Besonderes Verwaltungsrecht, V, Rn. 3.

14 Steiner, in: Steiner (Hrsg.), Besonderes Verwaltungsrecht, a.a.0.

15 Vgl. Runkel, in: Spannowsky u.a., Raumordnungsgesetz, § 1 Rn. $5 \mathrm{ff}$.

16 Durch Gesetz vom 1.9.2006 (BGBI. 2006 I, S. 2034ff.).

17 Wie weit dieses Abweichungsrecht reicht, ist noch in der juristischen Diskussion; eine Übersicht über den Meinungsstand gibt beispielsweise BMVBS/BBR (Hrsg.), Kompetenzen und Aufgaben der Raumordnung in der Gestaltung von Kulturlandschaften, BBR-Online-Publikation 19/2007, S. $19 \mathrm{ff}$.

18 Gesetz vom 22.12.2008 (BGBl. 2008 I, S. 2986ff.).

19 Nordrhein-Westfalen (NRW LplG v. 3.5.2005, GV. NRW, S. 430, zuletzt geändert durch Artikel 1 des Gesetzes vom 16.3.2010 (GV. NRW, S. 212)), Sachsen (SächsLPIG, Gesetz zur Raumordnung und Landesplanung des Freistaates Sachsen vom 11.6.2010, SächsGVBI. Nr. 8 vom 5.7.2010, S. 174), das Saarland (SLPG, Saarländisches Landesplanungsgesetz vom 18.11.2010, Amtsbl. I S. 2599) und Bayern (BayLplG, Bayerischsches Landesplanungsgesetz vom 25.6.2012, GVBI., S. 254) haben bereits Landesplanungsgesetze erlassen. 
Wesensmerkmale aufzeigen, die im Hinblick auf die Belange älterer Menschen von Bedeutung sind.

Das ROG enthält die Leitvorstellung einer „nachhaltigen Raumentwicklung“ in $\mathbf{8}$ $\S 1$ Abs. 2 ROG. Der Gesetzgeber hat diesen Begriff in Übereinstimmung mit dem international gebräuchlichen Begriff „sustainable development“ bewusst gewählt. ${ }^{20}$ Damit werden wirtschaftliche, soziale und ökologische Ansprüche an den Raum auf eine Stufe gestellt. ${ }^{21} \mathrm{Zu}$ den sozialen Ansprüchen an die Raumentwicklung wird man vor allem das Ziel der Generationengerechtigkeit zählen können. Raumordnerische Entscheidungen müssen so getroffen werden, dass sie die Entwicklungsmöglichkeiten künftiger Generationen nicht grundsätzlich behindern oder in Frage stellen. Diese Forderung ist heute an verschiedenen Stellen im Planungsrecht $\mathrm{zu}$ finden. Auch das Baugesetzbuch (BauGB) enthält für die Ortsebene die Forderung nach einer „nachhaltigen städtebaulichen Entwicklung“ (§1 Abs. 5 S. 1 BauGB). Konkrete Ableitungen lassen sich hieraus nicht treffen, so dass die Bedeutung der Leitvorstellung der „nachhaltigen Raumentwicklung“ weniger rechtlich, als vielmehr im „Ethos“ der Raumordnungsplaner zu sehen ist: sie formuliert den Anspruch, mit dem der Raumplaner an die Aufgabe herangeht. ${ }^{22}$

Die allgemeine Leitvorstellung der Raumordnung wird in §2 ROG durch „Grund- 9 sätze der Raumordnung“ ergänzt. Grundsätze sind Aussagen zur Raumentwicklung als Vorgaben für nachfolgende Abwägungs- und Ermessenentscheidungen (§3 Abs. 1 Nr. 3 ROG). Sie sind nicht nach Wichtigkeit geordnet, sondern beginnen allgemein und behandeln dann Einzelaspekte. ${ }^{23}$ Sie bilden kein widerspruchsfreies System, sondern eine Art Prüfliste für die Ermittlung der relevanten, bei der Planung zu berücksichtigenden Belange. Der zuständige Planungsträger hat dann im Rahmen seines Abwägungsspielraums die zu treffenden Maßnahmen zu bestimmen. Für den demographischen Wandel von Relevanz ist etwa die Forderung nach einer „,nachhaltigen Daseinsvorsorge“ und der Berücksichtigung „demographischer Herausforderungen“ (§2 Abs. 2 Nr. 1 ROG). Die schon in der Leitvorstellung angelegte Vorgabe der Nachhaltigkeit wird erneut aufgenommen: Die Gestaltungsmöglichkeiten der Raumordnung sind langfristig offen zu halten (§2 Abs. 2 Nr.1 S. 6 ROG). Es sollen möglichst durch die Zulassung von Raumnutzungen nicht Fakten in einer Weise geschaffen werden, die andere Möglichkeiten der Raumnutzung dauerhaft ausschließen. ${ }^{24} \S 2$ Abs. 2 Nr. 3 ROG betrifft insbesondere die Daseinsvorsorge. Sicherzustellen ist eine Grundversorgung und die Erreichbarkeit von Daseinsvorsorgeeinrichtungen für „alle

20 Runkel, in: Bielenberg u.a., Raumordnungsrecht, §1 Rn. 76; Runkel, in: Spannowsky u.a., Raumordnungsgesetz, §1 Rn. 96f.

21 Runkel, in: Spannowsky u.a., Raumordnungsgesetz, §1 Rn. 95.

22 Runkel, in: Bielenberg u.a., Raumordnungsrecht, L § 1 Rn. 73; ähnlich Runkel, in: Spannowsky u.a., Raumordnungsgesetz, §1 Rn. 42.

23 Runkel, in: Bielenberg u.a., Raumordnungsrecht, L § 2 Rn. 24.

24 Ähnlich Spannowsky, in: Spannowsky u.a., Raumordnungsgesetz, § 2 Rn. 62. 
Bevölkerungsgruppen“. Für die Erhaltung der Innenstädte und örtlichen Zentren als zentrale Versorgungsbereiche sind die räumlichen Voraussetzungen $\mathrm{zu}$ schaffen. Dies knüpft an die im Baugesetzbuch mehrfach angesprochene „Sicherung und Entwicklung zentraler Versorgungsbereiche“ ${ }^{25}$ an und ist dem demographischen und sozio-ökonomischen Wandel geschuldet. ${ }^{26}$ Die Raumordnung kann zu der primär städtebaulichen Aufgabe der Erhaltung der Innenstädte und örtlichen Zentren als zentrale Versorgungsbereiche jedoch nur insofern beitragen, als sie die räumlichen Voraussetzungen hierfür schafft, also z.B. außerhalb der zentralen Versorgungsbereiche keine großflächigen Einzelhandelsbetriebe zugelassen werden. ${ }^{27}$

10 Durch die Grundsätze der Raumordnung wird keine Verpflichtung aufgestellt, Anforderungen des demographischen Wandels in Raumordnungsplänen $\mathrm{zu}$ verankern. Erst recht gibt es keine Pflicht zur Veranlassung bestimmter Maßnahmen. Die Interessen der „Älteren“ sind grundsätzlich mit allen anderen öffentlichen Interessen gleichwertig.

11 Die Grundsätze des ROG werden ergänzt durch weitere Grundsätze in den Landesplanungsgesetzen (siehe $\S 3$ Abs. 1 Nr. 3 2. HS ROG; Wortlaut „insbesondere“ in $\S 2$ Abs. 2 ROG). Sie enthalten ähnliche weit formulierte Vorgaben wie $\S 2$ ROG, etwas stärker spezifiziert nach den Erfordernissen der jeweiligen Länder. Schließlich können Grundsätze auch in den Plänen, die auf Landesebene aufgestellt werden, enthalten sein. Die Landesplanung in den Ländern erfolgt zweistufig. Es ist ein landesweiter Raumordnungsplan aufzustellen (§ 8 Abs. 1 S. 1 Nr. 1 ROG), weiterhin Raumordnungspläne für die Teilräume ( $§ 8$ Abs. 1 S. 1 Nr. 2 ROG). Grundsätze finden sich vor allem auf der höheren Planungsstufe..$^{28}$ Die regionalen Teilpläne enthalten eher „Ziele“, also konkrete Raumnutzungsentscheidungen (§ 3 Abs. 1 Nr. 2 ROG). Welchen Inhalt solche Ziele haben, entscheiden die dafür zuständigen Planungsträger. Hierzu lassen sich kaum allgemeine Aussagen treffen, da sich die Anforderungen an die Gestaltung einer altersgerechten baulichen Umgebung, wie bereits angesprochen, stark unterscheiden.

\section{Die Umsetzung von Grundsätzen und Zielen der Raumordnung}

12 In den Raumordnungsplänen kommt es zur Schaffung von „Zielen der Raumordnung“ im Sinne von verbindlichen Raumnutzungsentscheidungen. Dies sind die

25 Z.B. in § 1 Abs. 6 Nr. 4 BauGB, § 2 Abs. 2 S. 2 BauGB, § 9 Abs. 2a BauGB sowie § 34 Abs. 3 BauGB; Söfker, UPR 2009, S. 161, 162.

26 Spannowsky, in: Spannowsky u.a., Raumordnungsgesetz, § 2 Rn. 91.

27 Runkel, in: in: Bielenberg u.a., Raumordnungsrecht, L § 2 Rn. 139.

28 Als Beispiel: Bayerisches Landesentwicklungsprogramm v. 1.9.2006, B VI 2.2: „Die Belange alter Menschen und Menschen mit Behinderung sind im Städtebau und bei der Schaffung von Wohnraum zu berücksichtigen.“ Dies ist mit „(G)“ für „Grundsatz“ gekennzeichnet. 
maßgeblichen Vorgaben für die weitere Ordnung des Planungsgeschehens entsprechend den Vorstellungen der überörtlichen Planungsebene. „Ziele“ haben jedoch keinen einer Rechtsnorm vergleichbaren allgemeinen Geltungsanspruch. Ihre Bindungswirkung ist nach §4 ROG von verschiedenen Faktoren abhängig. Im Grundsatz sind nur öffentliche Stellen an die Ziele gebunden. Private sind - vereinfacht gesprochen - nur dann von den Zielen betroffen, wenn die Verwaltungsbehörden im Rahmen von Verwaltungsentscheidungen Entscheidungsspielräume haben (Ermessens- oder Abwägungsentscheidungen). Grundsätze der Raumordnung sind im Rahmen solcher Entscheidungen ebenfalls als „Abwägungsmaterial“ zu beachten.

Vor allem trifft die Bindung durch Ziele der Raumordnung die Gemeinden bei $\mathbf{1 3}$ ihrer Bauleitplanung ( $§ 1$ Abs. 4 BauGB). Sie müssen vorhandene Bauleitpläne (also Bebauungspläne und Flächennutzungspläne) anpassen bzw. beim Neuerlass solcher Pläne die Ziele der Raumordnung beachten. Konfliktträchtig sind vor allem Fälle, in denen den Gemeinden die Ansiedlung großflächiger Einzelhandelsbetriebe durch Festlegungen in der Raumordnung untersagt werden soll. Insoweit besteht ein gewisser Bezug zum „Recht der Älteren“. Solche Festsetzungen dienen in der Regel dem Schutz von anderen Versorgungsbereichen, die möglicherweise gerade für ältere Menschen besser erreichbar sind (Schutz einer verbrauchernahen Versorgung).

\section{Belange der Älteren im Städtebaurecht}

\section{Die Belange der Älteren in der Bauleitplanung}

Das Recht der Bauleitplanung ist von ähnlichen Prinzipien geprägt wie das Raum- 14 ordnungsrecht. Das Baugesetzbuch nennt zunächst allgemeine Leitvorstellungen, die bei der Aufstellung von Bauleitplänen zu verfolgen sind (§1 Abs. 5 BauGB). Auch das BauGB verlangt in $\S 1$ Abs. 5 S. 1 BauGB in sehr allgemeiner Weise die Beachtung „sozialer Anforderungen“ (neben wirtschaftlichen und ökologischen Belangen), ebenso die „Verantwortung gegenüber künftigen Generationen“. Diese Leitvorstellungen werden in $\S 1$ Abs. 6 BauGB um eine lange Liste von abwägungserheblichen Gesichtspunkten ergänzt, u.a. die Berücksichtigung von Wohnbedürfnissen der Bevölkerung ( $§ 1$ Abs. 6 Nr. 1 BauGB), die Beachtung der sozialen und kulturellen Bedürfnisse, auch der ,alten und behinderten Menschen“ (§ 1 Abs. 6 Nr. 3 BauGB), sowie die Erhaltung und Entwicklung zentraler Versorgungsbereiche ( $\S 1$ Abs. 6 Nr. 4 BauGB). Die Belange einer älter werdenden Bevölkerung sind im Rahmen der Abwägung bei der Aufstellung der Bauleitpläne zu berücksichtigen ( 11 Abs. 7 BauGB). Sie erhalten keinen stärkeren, aber auch keinen geringeren Rang als andere öffentliche Interessen auch.

Der Umweltschutz hat in den letzten Jahrzehnten als Belang im Rahmen der 15 Bauleitplanung besondere Bedeutung erlangt. Insoweit sind für die Abwägung be- 
sondere Vorschriften getroffen worden (§ 1a BauGB). Der Belang Umweltschutz wird zudem prozedural besonders hervorgehoben. Es ist eine eigene Umweltprüfung durchzuführen, die Ergebnisse sind in einem Umweltbericht zusammenzufassen (§ 2 Abs. 4 BauGB). Dadurch ist die Bauleitplanung derzeit stark umweltzentriert, eine Reaktion auf die Vernachlässigung der Umweltbelange in den 1960er und 1970erJahren. Für eine tendenziell stärkere Berücksichtigung der Interessen älterer Menschen könnten diese Vorschriften Vorbildfunktion haben, wenn sich das Bedürfnis ergeben sollte, vergleichbar der Berücksichtigung von Umweltbelangen, auch die Belange älterer Menschen besonders abzusichern. Die hervorgehobene Nennung von Belangen im Rahmen des $\S 1$ Abs. 6 BauGB führt erfahrungsgemäß, wenn überhaupt, nur zu geringen Effekten für die Planung. Erfolgreicher ist die Prozeduralisierung, die die Planer ausdrücklich dazu zwingt, sich mit den Folgen für einen speziellen Belang in besonderer Weise auseinanderzusetzen. Dies sind jedoch rein rechtspolitische Überlegungen. Die Interessen der Älteren sind derzeit gleichwertig zu allen anderen Interessen. Da, anders als die Umwelt, ältere Menschen über demokratische Mitwirkungsrechte verfügen, ist eine Übertragung von Instrumenten aus dem Umweltschutzrecht möglicherweise auch entbehrlich.

\section{Die Belange älterer Menschen im sonstigen allgemeinen Städtebaurecht}

16 Die Interessen älterer Menschen an geeigneten Infrastruktureinrichtungen sind vielfach deckungsgleich mit den Bedürfnissen anderer Bevölkerungsgruppen. Auch Familien mit Kindern benötigen einen funktionierenden öffentlichen Personennahverkehr, medizinische Versorgung und in der Nähe gelegene Einkaufsmöglichkeiten. ${ }^{29}$ Deshalb sind die Normen des Städtebaurechts, die beispielsweise dem Schutz von zentralen Versorgungseinrichtungen dienen, auch, aber nicht nur für ältere Menschen von Bedeutung. So hat der Gesetzgeber in den letzten Jahren erhebliche Anstrengungen unternommen, um eine verbrauchernahe Versorgung der Bevölkerung sicherzustellen. Vor allem $\S 9$ Abs. 2a BauGB bietet die Möglichkeit, im sog. Innenbereich Festsetzungen zu treffen, die beispielsweise den unkontrollierten Aufbau von Einzelhandelsangeboten regulieren. ${ }^{30}$ Damit soll erreicht werden, dass funktionierende Versorgungsbereiche gegen eine möglicherweise ruinöse Konkurrenz an anderer Stelle abgeschirmt werden.

$17 \S 34$ Abs. 3 BauGB beschränkt ebenfalls die Zulässigkeit von baulichen Anlagen in den Fällen, in denen schädliche Auswirkungen auf zentrale Versorgungsbereiche und damit auf die verbrauchernahe Versorgung befürchtet werden. ${ }^{31}$ Eine ähnliche

29 Siehe dazu allgemein Schmitz, ZfBR 2007, S. 532ff.

30 Näher zu § 9 Abs. 2a BauGB: Klinge, BauR 2008, S. 770ff.; Reidt, BauR 2007, S. 2001ff.

31 Vgl. dazu Janning, BauR 2005, S. 1723ff.; Reichelt, BauR 2006, S. 38ff.; Söfker, in: Ernst/Zinkahn/Bielenberg/Krautzberger, BauGB, § 34 Rn. $83 f f$. 
Funktion haben $\S 1$ Abs. 5 und 9 sowie $\S 11$ Abs. 3 BauNVO für den überplanten Bereich. Die Kommunen als zuständige Träger der Bauleitplanung haben die sehr weitreichende Möglichkeit, Verkaufsbeschränkungen etwa für Gewerbe- und Industriegebiete festzusetzen, um die vorhandenen Geschäfte abzusichern. Einkaufszentren und großflächige Einzelhandelsbetriebe ${ }^{32}$ werden von $\S 11$ Abs. 3 BauNVO im Grundsatz nur noch in Kerngebieten und von der Gemeinde extra geplanten Sondergebieten zugelassen. Der insbesondere für ältere Menschen ungünstigen Tendenz, auf der grünen Wiese Einkaufsmöglichkeiten bereitzustellen, die zu einem Sterben kleinerer Geschäfte in den Innenstädten führt, soll damit entgegengewirkt werden.

Das Bauplanungsrecht ist bisher an kaum einer Stelle wegen des demographi- 18 schen Wandels ausdrücklich geändert worden. Eine Ausnahme findet sich in $\S 3$ Abs. 4 BauNVO. In einer Novelle von 1990 wurde festgelegt, dass zu den Wohngebäuden auch solche gehören, die ganz oder teilweise der Betreuung und Pflege ihrer Bewohner dienen. ${ }^{33}$ Damit wird der Begriff des Wohnens teilweise erweitert, zu Lasten der „Wohnruhe“ in den sog. reinen Wohngebieten, denn ein höheres Störungspotential (vermehrter $\mathrm{Zu}$ - und Abfahrtsverkehr) ist offensichtlich. Reine Betreuungs- oder Pflegeeinrichtungen sind allerdings in reinen Wohngebieten nach wie vor nicht zulässig. ${ }^{34}$ Es muss ein Mindestmaß an „selbstbestimmtem Wohnen“ vorliegen, ${ }^{35}$ ein gewisser „Kernbestand an Häuslichkeit“ gegeben sein. ${ }^{36}$ Dann kann ein Gebäude selbst bei vollständiger Betreuung und Pflege als Wohngebäude zu werten sein (z.B. für Demenzkranke). ${ }^{37}$ Rehabilitationseinrichtungen hingegen, bei denen der gesundheitliche Zweck im Vordergrund steht, wird man nicht als Wohngebäude ansehen können. Hierbei handelt es sich um Anlagen für gesundheitliche und soziale Zwecke, die in reinen Wohngebieten weder zulässig noch ausnahmsweise zulässig sind..$^{38}$ Die Abgrenzung zwischen „Wohnen“ und „Einrichtungen für gesundheitliche und soziale Zwecke“ kann schwierig sein. ${ }^{39}$ Von „Wohnen“ kann nicht mehr die Rede sein, wenn die Anlage mit ihren durch die Funktionsfähigkeit bedingten Ansprüchen die Lebensführung des Bewohners bestimmt..$^{40}$

32 Von einem großflächigen Einzelhandelsbetrieb geht man aus, wenn die Verkaufsfläche mehr als 700 qm beträgt.

33 Ausführlicher dazu Bunzel/Löhr, ZfBR 2000, S. 307, 311.

34 Siehe auch Stock, in: König/Roeser/Stock, BauNVO, § 3 Rn. 30 m.w. Nachw.

35 Stock, in: König/Roeser/Stock, BauNVO, § 3 Rn. 30.

36 Stock, in: König/Roeser/Stock, BauNVO, § 3 Rn. 30.

37 Vgl. BVerwG, NVwZ 1996, 893, 894; v. Franckenstein, ZfBR 2008, S. 763, 767.

38 Siehe v. Franckenstein, ZfBR 2008, S. 763, 767.

39 Zur Frage, wann sich eine Nutzung noch als „Altenwohnheim“ darstellt, siehe BayVGH, BayVBI. 2012, 86ff.

40 v. Franckenstein, ZfBR 2008, S. 763, 767. 


\section{Demographischer Wandel und besonderes Städtebaurecht}

19 Der demographische Wandel und der steigende Altersdurchschnitt können zu städtebaulichen Problemen führen, deren Lösung ein abgestimmtes Instrumentarium von planerischen und finanziellen Maßnahmen erfordert. Ansätze dafür finden sich im sog. besonderen Städtebaurecht. $\mathrm{Zu}$ nennen sind insbesondere die Vorschriften über den Stadtumbau ( $\S \S 171 \mathrm{a}-171 \mathrm{~d}$ BauGB). ${ }^{41}$ Mit Stadtumbaumaßnahmen soll auf einen städtebaulichen Funktionsverlust reagiert werden (§ 171a Abs. 2 BauGB). Ein vom Gesetzgeber hervorgehobenes Problemfeld ist ein dauerhaftes Überangebot an baulichen Anlagen insbesondere für Wohnzwecke (§171a Abs. 2 S. 2 BauGB). Maßnahmen, die in solchen Fällen in Betracht kommen, bestehen unter anderem darin, dass die vorhandenen Anlagen neuen Nutzungen zugeführt (§171a Abs. 3 Nr. 4 BauGB) oder zurückgebaut werden ( $§ 171$ a Abs. 3 Nr. 5 BauGB). Ein Gebiet für den Stadtumbau kann durch Satzung der Gemeinden festgelegt werden (§ 171d BauGB). Vorrangig sollen allerdings Maßnahmen mit den Eigentümern auf vertraglicher Grundlage erfolgen (§ 171c BauGB).

20 Hinzuweisen ist aber darauf, dass das Problem des Stadtumbaus nicht nur vor dem Hintergrund des demographischen Wandels gesehen werden kann. Der Rückbau etwa von Plattenbausiedlungen in den neuen Bundesländern ist in den letzten Jahren auch deshalb erforderlich geworden, weil die Bevölkerung wegen Wegzugs abnimmt. ${ }^{42}$

21 Eine weitere Regelung mit Bezug zum demographischen Wandel ist die Regelung über die soziale Stadt nach $\S 171$ e BauGB. Allgemein geht es bei dieser Bestimmung um das Problem der Segregation. ${ }^{43}$ Mit Hilfe eines von der Gemeinde initiierten Entwicklungskonzepts sollen soziale, wirtschaftliche und politische Potentiale formuliert und gefördert werden (§ 171e Abs. 4 BauGB). Die Beteiligten sollen in geeigneter Weise einbezogen werden ( $\S 171$ e Abs. 5 BauGB). Probleme der Segregation können durch den demographischen Wandel entstehen, etwa wenn sich Stadtquartiere bilden, in denen überwiegend ältere Leute wohnen. Typisch für das gesamte Planungsrecht ist aber erneut der Befund, dass die Alterung der Bevölkerung nur eine mögliche Ursache für Fehlentwicklungen ist. Auch andere Gründe können eine Segregation zur Folge haben. So können auch Migrationsbewegungen zu städtebaulichen Fehlentwicklungen führen, etwa wenn bestimmte Quartiere nur noch von Migranten oder einer bestimmten Gruppe von Migranten bewohnt werden.

41 Hierzu und zum weiteren siehe Kersten, Die Verwaltung 40 (2007), S. 309, 327ff.; ders., in: Voßkuhle/Schuppert (Hrsg.), Governance von und durch Wissen, S. 190, 196ff.; ders., RuR 2006, S. $245 \mathrm{ff}$.

42 Siehe Mitschang/Roeper, ZfBR 2011, S. $10 \mathrm{ff}$.

43 Siehe auch dazu Kersten, Die Verwaltung 40 (2007), S. 309, $327 f f$. 


\section{Belange der Älteren im Bauordnungsrecht}

Die Anforderungen an einzelne Gebäude unabhängig von ihrer planungsrechtlichen 22 Einordnung sind Gegenstand des Bauordnungsrechts. ${ }^{44}$ Es ist in den Bauordnungen der Länder geregelt, die sich inhaltlich in den Grundzügen weitgehend entsprechen. ${ }^{45}$ Eine spezifische Ausrichtung auf altersgerechtes Bauen ist allerdings im Bauordnungsrecht nicht $\mathrm{zu}$ finden. Auch in diesem Bereich gibt es nur vereinzelte Bestimmungen, die einen speziellen Bezug zum Recht der Älteren haben. ${ }^{46}$

Besonders erwähnenswert ist die Regelung über barrierefreies Bauen (Art. 48 BayBO). Bei Gebäuden mit mehr als zwei Wohnungen müssen die Wohnungen eines Geschosses barrierefrei erreichbar sein, gegebenenfalls müssen die Wohn- und Schlafräume, eine Toilette, ein Bad, die Küche sowie ein Raum mit Anschlussmöglichkeit für eine Waschmaschine mit einem Rollstuhl erreichbar und barrierefrei nutzbar sein (Art. 48 Abs. 1 S. 2 BayBO). Weitere Anforderungen gelten für bauliche Anlagen, die öffentlich zugänglich sind (Art. 48 Abs. 2 BayBO). Sie müssen barrierefrei zugänglich und ohne fremde Hilfe in der allgemein üblichen Weise zweckentsprechend nutzbar sein.

Diese Anforderungen gelten allerdings - wie überwiegend alle neu geschaffe- 24 nen baurechtlichen Regelungen - nur für Neubauten bzw. im Falle der Änderung oder Nutzungsänderung von baulichen Anlagen. Sie betreffen also nicht den Altbestand.

\section{Fazit}

Der demographische Wandel ist auch für das Bau- und Planungsrecht eine Heraus- 25 forderung. Dieses Rechtsgebiet ist aber ohnehin so angelegt, dass divergierende Interessen einem Ausgleich zugeführt werden, insbesondere über das bei der Aufstellung von Plänen dominierende Abwägungsgebot. Es ist deshalb sowieso möglich, dass die Planungsträger aufgrund von neuen gesellschaftlichen Entwicklungen entsprechende Entscheidungen auf planungsrechtlicher Ebene treffen. Es gibt deshalb vor allem im Planungsrecht keinen signifikanten gesetzgeberischen Anpassungsbedarf im Hinblick auf das „Recht der Älteren“. Im Bauordnungsrecht ist das Ziel eines altersgerechten Wohnens insbesondere durch die Bestimmung über barrierefreies Bauen im Wesentlichen erreicht.

\footnotetext{
44 Zur Unterscheidung von Bauplanungs- und Bauordnungsrecht allgemein Manssen, in: Becker/Heckmann/Kempen/Manssen, Öffentliches Recht in Bayern, 4. Teil Rn. $3 f f$. 45 Im Folgenden werden Gesetzeszitate nach der Bayerischen Bauordnung vorgenommen, die bundesweit stets eine Art Vorreiterrolle für neue Entwicklungen spielt.

46 Schröer/Kulick, NZBau 2011, S. $90 \mathrm{ff}$.
} 
Bereitgestellt von | De Gruyter / TCS Angemeldet 